OPEN ACCESS

Edited by:

Chen Qingfeng,

Guangxi University, China

Reviewed by:

Ximiao He,

Huazhong University of Science and Technology, China

Jinping Liu,

University of Pennsylvania,

United States

Huiyu Zhou,

University of Leicester,

United Kingdom

${ }^{*}$ Correspondence:

Yuanliang Xie

136641161@qq.com

Qiuyan Wang

qiuyanwang510@yahoo.com

Zhong Tang

18978868502@qq.com

${ }^{\dagger}$ These authors have contributed equally to this work

Specialty section:

This article was submitted to

Computational Genomics,

a section of the journa

Frontiers in Genetics

Received: 15 April 2021

Accepted: 11 June 2021

Published: 02 July 2021

Citation:

Wang $Z$, Wang $X$, Wang $Y$, Tang S, Feng $C$, Pan L, Lu Q, Tao $Y$, Xie Y, Wang $Q$ and Tang $Z$ (2021) Transcriptomic Analysis of Gene Networks Regulated by U11 Small

Nuclear RNA in Bladder Cancer.

Front. Genet. 12:695597.

doi: 10.3389/fgene.2021.695597

\section{Transcriptomic Analysis of Gene Networks Regulated by U11 Small Nuclear RNA in Bladder Cancer}

\author{
Zhenxing Wang ${ }^{1,2,3 \dagger}$, Xi Wang ${ }^{1,2 \dagger}$, Yaobang Wang ${ }^{1,2 \dagger}$, Shaomei Tang ${ }^{4}$, Chao Feng ${ }^{1,2}$, \\ Lixin Pan ${ }^{1,2}$, Qinchen Lu ${ }^{1,2}$, Yuting Tao ${ }^{1,2}$, Yuanliang Xie ${ }^{1,2,5 *}$, Qiuyan Wang ${ }^{1,2 *}$ and \\ Zhong Tang $1,2,6 *$
}

\begin{abstract}
' Center for Genomic and Personalized Medicine, Guangxi Medical University, Nanning, China, ${ }^{2}$ Guangxi Key Laboratory for Genomic and Personalized Medicine, Guangxi Collaborative Innovation Center for Genomic and Personalized Medicine, Nanning, China, ${ }^{3}$ Department of Clinical Laboratory, The First Affiliated Hospital of Guangxi Medical University, Nanning, China, ${ }^{4}$ Department of Gastroenterology, The First Affiliated Hospital of Guangxi Medical University, Nanning, China, ${ }^{5}$ Department of Urology, The Affiliated Cancer Hospital of Guangxi Medical University, Nanning, China, ${ }^{6}$ School of Information and Management, Guangxi Medical University, Nanning, China
\end{abstract}

Small nuclear RNA is a class of non-coding RNA that widely exist in the nucleus of eukaryotes. Accumulated evidences have shown that small nuclear RNAs are associated with the regulation of gene expression in various tumor types. To explore the gene expression changes and its potential effects mediated by U11 snRNA in bladder cancer cells, U11 snRNA knockout and overexpressed cell lines were constructed and further used to analyze the gene expression changes by RNA sequencing. The differentially expressed genes were found to be mainly enriched in tumor-related pathways both in the U11 knockout and overexpression cell lines, such as NF-kappa B signaling pathway, bladder cancer and PI3K-Akt signaling pathway. Furthermore, alternative splicing events were proposed to participate in the potential regulatory mechanism induced by the U11 knockout or overexpression. In conclusion, U11 may be involved in the regulation of gene expression in bladder cancer cells, which may provide a potentially new biomarker for clinical diagnosis and treatment of bladder cancer.

Keywords: U11 small nuclear RNA, bladder cancer, T24, transcriptomic analysis, gene networks

\section{INTRODUCTION}

Bladder cancer is one of the most common urological cancers, ranking ninth among all malignant tumors worldwide and sixth among men (Ploeg et al., 2009; Bray et al., 2018). In the United States, bladder cancer ranks fourth among all malignant tumors, with 74,000 new cases of bladder cancer in 2015, including 56,320 males and 17,680 females, and the estimated fatal cases were 16,000, including 11,510 males and 4,490 females. In South Asia and Western Asia, the incidence and mortality of bladder cancer also rank top among all malignant tumors (Salim et al., 2010). Although the incidence and mortality of bladder cancer in China are lower than the world average level, there is a trend of increasing incidence in some cities (Feng et al., 2019), which seriously threatens the survival health and life quality of patients. Therefore, it is of great significance to study the mechanism of the occurrence and development of bladder cancer, and further to improve the diagnosis and treatment rate of bladder cancer. 
Cajal bodies, also known as coiled bodies, were first discovered in the nucleus of nerve cells by Ramony Cajal in 1903 (Hebert, 2010). Cajal bodies are widely found in the nucleus of higher eukaryotes, and their number and size can vary with species. Cajal bodies are more abundant in somatic cells of differentiated tissues and some cells with higher metabolic activity, such as muscle, neurons, and tumor cells (Hearst et al., 2009; Machyna et al., 2013). At the same time, the numbers and sizes of Cajal bodies are related to the cell cycle, and their numbers often reach the maximum in the $\mathrm{G} 1 / \mathrm{S}$ phase. In general, Cajal bodies depolymerize in the $\mathrm{M}$ phase, and the reassembly process depends on the level of gene transcription and the rate of proliferation of the cell. Relevant studies have shown that there are a large number of factors involved in the splicing of mRNA precursors, rRNA precursor processing, histone pre-mRNA $3^{\prime}$ end processing and telomere maintenance in the components of Cajal bodies, suggesting that Cajal bodies may be a site for the assembly and function of ribonucleoprotein (RNP) (Hebert, 2013). Cajal bodies can also serve as a platform for effective modification responses in transcriptionally active cells requiring high levels of RNP, such as neuronal cells and tumor cells. Studies have found that zebrafish embryos are unable to complete embryonic development due to the lack of coilin and Cajal bodies. The depletion of coilin and Cajal bodies was mainly characterized by deficits in snRNP biogenesis and expression of spliced mRNA, while mature snRNPs can partially rescue embryonic lethal phenotypes (Strzelecka et al., 2010).

At present, more than dozens of proteins have been found to localize or interact with Cajal bodies (Machyna et al., 2013). Coilin is recognized as a marker protein and a major component of Cajal bodies (Andrade et al., 1991). One of the most significant structural features of Cajal bodies is the accumulation of a large number of non-coding small RNAs, which include U1, U2, U3, U4, U5, U6, U7, U8, U11, U12, U13, U14, U64, U6atac, and U87 scaRNA, etc. These small non-coding RNAs were once thought to be mainly involved in post-transcriptional modification of RNA. However, with the development of highdepth sequencing technology and bioinformatics technology, the functions of these small non-coding RNAs have been further recognized and understood. Studies have found that they may be involved in gene expression, genome structure organization, and other functions (Lui and Lowe, 2013). In recent years, noncoding RNAs have attracted much attention as a special way of gene expression regulation.

Our previous studies innovatively proposed the notion that Cajal bodies can be simultaneously linked with multiple small molecule RNA gene loci to form gene clusters, using Hela cell models and six-color microscopes detection systems (Wang et al., 2016). Moreover, we found that this gene cluster is not formed randomly but is a specific spatial structure of long-distance interactions. U1, U2, U3, U4, U5, and U11 are small non-coding RNA genes enriched in Cajal bodies, U87 scaRNA is small Cajal body-specific RNA gene, Histone cluster 2 and Histone H3F3 are histone small RNA genes. Among them, U1, U2, U3, U4, U5, and U11 are small non-coding RNA genes enriched in Cajal bodies. Using chromatin spatial conformation capture technology, it was found that small nuclear RNA mediates the formation of long-distance chromatin interactions (Wang et al., 2016). U11 (RNU11) is probably the most significant small nuclear RNA because its expression is extremely high expressed in rapidly growing tumor cells and very low expressed in slow-growing primary cells. Thus, the above studies provided the evidence that U11 small nuclear RNA plays a role in regulating the spatial structure of chromatin and may be of great significance in the development of tumors.

Interestingly, we found Cajal bodies were aberrantly activated in T24 bladder cancer cell lines and the increased numbers and sizes of Cajal bodies were displayed in two highly invasive and metastatic T24-SLT and T24-FL cell lines. Given that U11 is one of the most crucial snRNAs located in Cajal bodies, we speculated that U11 might play an important role in the gene expression of bladder cancer cells. In this study, the in vitro cell models with U11 knockout and overexpression were firstly constructed in T24 bladder cancer cell lines and further used to analyze the gene expression changes using RNA-sequencing technology. The differentially expressed genes were found to be mainly enriched in tumor-related pathways both in the U11 knockout and overexpression groups. Notably, alternative splicing events were innovatively proposed to participate in the potential regulatory mechanism induced by U11 knockout or overexpression. Taken together, our study innovatively elucidated that U11 may play the critical role in the regulation of gene expression in bladder cancer cells, which may provide a potentially new biomarker for clinical diagnosis and treatment of bladder cancer.

\section{RESULTS}

\section{Cajal Body-Related snRNA U11 Affects the Occurrence and Development of Bladder Cancer by Regulating Differently Expressed Genes}

By using immunofluorescence (IF) staining, we unexpectedly found Cajal bodies were aberrantly activated in T24 bladder cancer cell lines. More interestingly, the increased numbers and sizes of Cajal bodies were displayed in two highly invasive and metastatic T24-SLT and T24-FL cell lines (Nicholson et al., 2004; Jeppesen et al., 2014) (Figure 1A). Given that U11 is one of the most crucial snRNAs located in Cajal bodies, the in vitro cell models with U11 knockout and overexpression were successfully established in T24 cell lines. The knockout and overexpression efficiency of U11 in T24 cell lines were confirmed by real-time quantitative PCR, the expression level of U11 in U11-KO cell line was significantly decreased, and that of U11-KI cell line was significantly overexpressed compared to T24 WT cell line (Figure 1B). Moreover, MTT assay revealed that the cell proliferation ability of T24 WT cell line was significantly higher than that of the U11-KO cell line $(P<0.001$, Figure 1C). The U11 knockout and U11 overexpression groups were used as experimental groups, and gene differences were analyzed by comparing with the control group. A total of 2,756 differentially expressed genes in the U11 knockout group were obtained, including 1,464 up-regulated and 1,292 down-regulated 


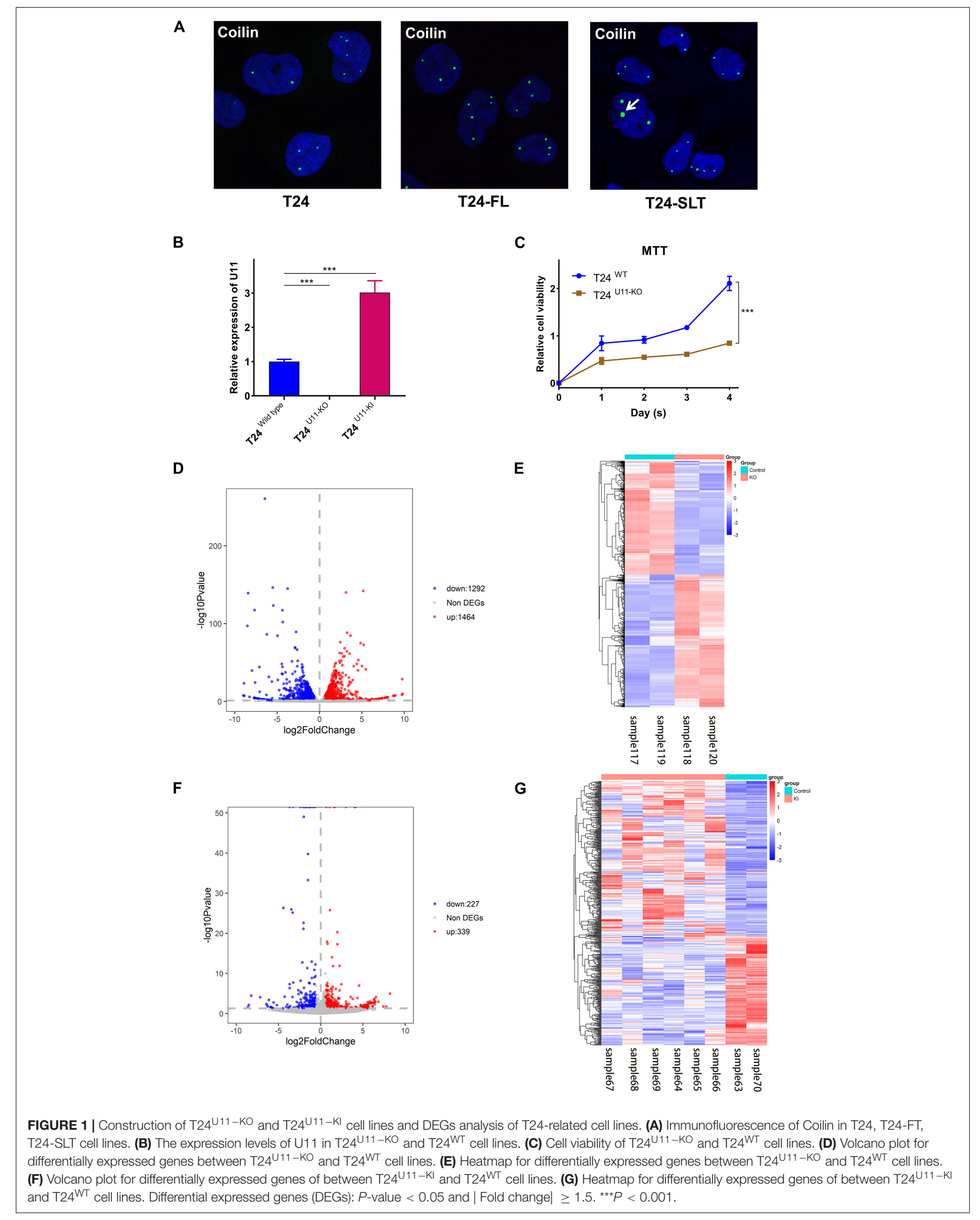


(Figures 1D,E); In addition, there were 566 differentially expressed genes in the U11 overexpression group, including 339 up-regulated and 227 down-regulated (Figures 1F,G). Pathway enrichment analysis by clusterProfiler $\mathrm{R}$ package showed that the up-regulated genes obtained by overexpressing U11 were mainly enriched in regulation of mast cell degranulation, chemokine activity, NF-kappa B signaling pathway, TNF signaling pathway, and Bladder cancer, etc. (Figures 2A,B). The down-regulated genes obtained by overexpressing U11 were mainly enriched in integral component of lumenal side of endoplasmic reticulum membrane, cellular response to type I interferon, defense response to virus, Allograft rejection and Antigen processing and presentation, etc. (Figures 2C,D). Interestingly, we found that the enrichment pathways of down-regulated genes in the U11 knockout group were similar to those of up-regulated genes in the U11 overexpression group. The pathways of downregulated genes in U11 knockout group were mainly enriched in $\mathrm{T}$ cell apoptotic process, cytokine receptor binding, TNF signaling pathway, and NF-kappa B signaling pathway, etc. (Figures 2G,H). The pathways of up-regulated genes in U11 knockout group were mainly enriched in laminin binding, fibronectin binding, cell-substrate adhesion, Proteoglycans in cancer, and PI3K-Akt signaling pathway (Figures 2E,F). Among them, Figure 3 showed the PI3K-Akt signaling pathway and the genes involved in this pathway.

Subsequently, we used the up-regulated genes in the U11 overexpression group and the down-regulated genes in the U11 knockout group for intersection analysis, as well as the downregulated genes in the U11 overexpression group and the upregulated genes in the U11 knockout group for intersection analysis. We found that there were 93 intersecting genes in the two intersecting groups (Figures 4A,B). Using these genes for protein interaction network analysis, we found that the hub genes were mainly CXCL2, CXCL3, CXCL6, CXCL8, and other CXCL gene families (Figure 4C). Interestingly, we found that the expression of CXCL2, CXCL3, CXCL6, CXCL8, and other CXCL gene families was significantly up-regulated in the U11 overexpression group, while the expression of these genes was significantly down-regulated in the U11 knockout group (Figures 4D,E). The intersecting genes of up-regulated genes in U11 knockout group and down-regulated genes in U11 overexpression group were mainly enriched in regulation of cell adhesion mediated by integrin, fibroblast migration and regulation of lipolysis in adipocytes (Figure $4 \mathbf{F}$ ), and the intersecting genes of down-regulated genes in U11 knockout group and up-regulated genes in U11 overexpression group were mainly enriched in chemokine activity, response to lipopolysaccharide, neutrophil migration, NF-kappa B signaling pathway, TNF signaling pathway, and transcriptional misregulation in cancer (Figure 4G).

\section{U11 Alters Gene-Splicing Events and Gene Expression}

We further performed alternative splicing analysis and found that a total of 4,023 genes in the U11 overexpression group had significant differential alternative splicing events. Among them, exon skipping (SE) was the most frequent event, while intron retention (RI) was the least frequent event, and 316 genes were simultaneously exposed to five alternative splicing events (Figure 5A). Similarly, 4,774 genes were found to have significant differential alternative splicing events in the U11 knockout group, with exon skipping events occurring most frequently (Figure 5B).

Next, we intersected genes with differentially alternative splicing events and differentially expressed genes (Figure 6A). Seventy-one intersecting genes were obtained in the U11 overexpression group. Among them, murine double minute 2 (MDM2) gene had one exon skip and one mutually exclusive exon event, and the gene expression increased about 3.5-fold, and TGFB2 gene had one exon skip, and the gene expression decreased 2.1-fold (Figures 6B,E). Notably, 648 intersecting genes in the U11 knockout group, which were mainly enriched in pathways such as NF-kappa B signaling pathway and TNF signaling pathway (Figure 6C). The results of protein interaction network analysis of these intersecting genes also showed that the hub genes mainly included genes such as TIMP1, FN1, and RPL22L1 (Figure 6D). Intriguingly, FN1 gene had multiple alternative $3^{\prime}$ splice site (A3SS) events, one mutually exclusive exon event, four exon skipping events, and the level of mRNA expression increased 2.9-fold. TIMP1 gene had only one exon skipping event, and the level of mRNA expression increased 3.5fold. RPL22L1 gene had one exon skipping events, and the level of expression decreased 1.7-fold (Figures 6B,E).

Given that the alternative splicing events of TIMP1, FN1, and RPL22L1 have been widely reported to participate in several biological processes (Usher et al., 2007; Lopez-Mejia et al., 2013; Liang et al., 2019), we therefore validated the AS events of the genes described above, using PCR and gel electrophoresis. We initially examined three typical exons skipping of FN1 (EDA, EDB, and IIICS) as previously reported (Lopez-Mejia et al., 2013), but no remarkably alternative splice events were detected in FN1 gene. The full-length TIMP1 transcript was then detected by the forward primer located in exon 1 combined with the reverse primer located in exon 6. Intriguingly, the band of full-length TIMP1 in $\mathrm{T} 24^{\mathrm{U} 11-\mathrm{KO}}$ cell line was observed to shift down a weak distance less than an exon, compared with T24 ${ }^{\mathrm{WT}}$ cell line. What' more, as the gel picture shown and the gray arrow indicated in Figure 6F, one indistinctly visible band appeared below the major band in $\mathrm{T} 24^{\mathrm{U} 11-\mathrm{KO}}$ cell line, but not in T24 ${ }^{\mathrm{WT}}$ cell line, suggesting a potential AS event of TIMP1 after U11 knockout. Three exons of TIMP1 gene were further examined the alternative splicing events, respectively. However, no significant alteration of splicing patterns was observed in the TIMP1-1, TIMP1-2, and TIMP1-3 segments because of the rare frequency and low abundance of the exon skipping (Figure 6F). All these results indicated that a link between alternative splicing regulated by $\mathrm{U} 11$ and bladder carcinogenesis.

\section{FN1 and RPL22L1 May Be a Prognostic Marker for Bladder Cancer}

To further investigate whether hub genes have an impact on the prognosis of bladder cancer, we performed survival analysis of CXCL8, MDM2, TGFB2, FN1, TIMP1, and RPL22L1, and 


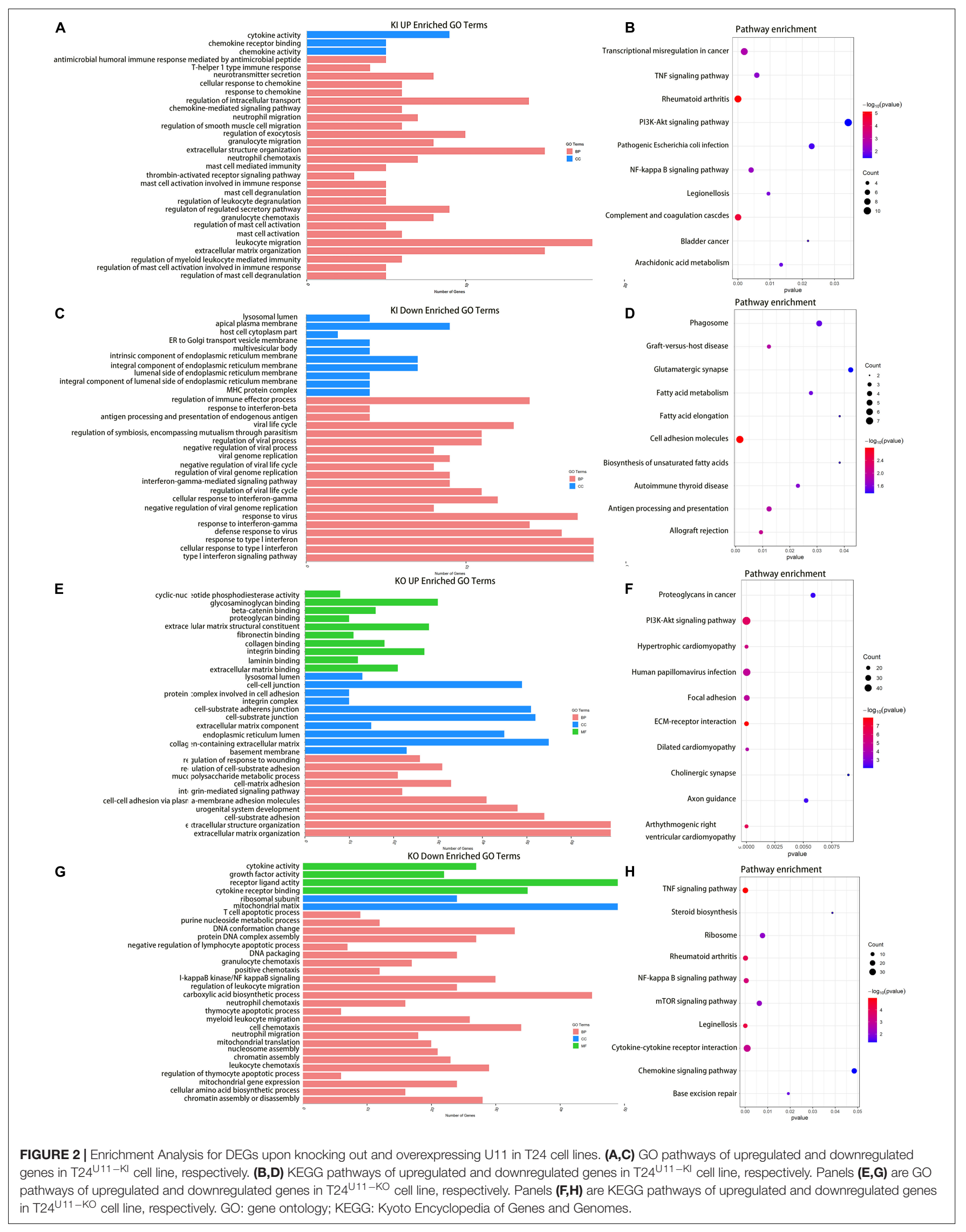



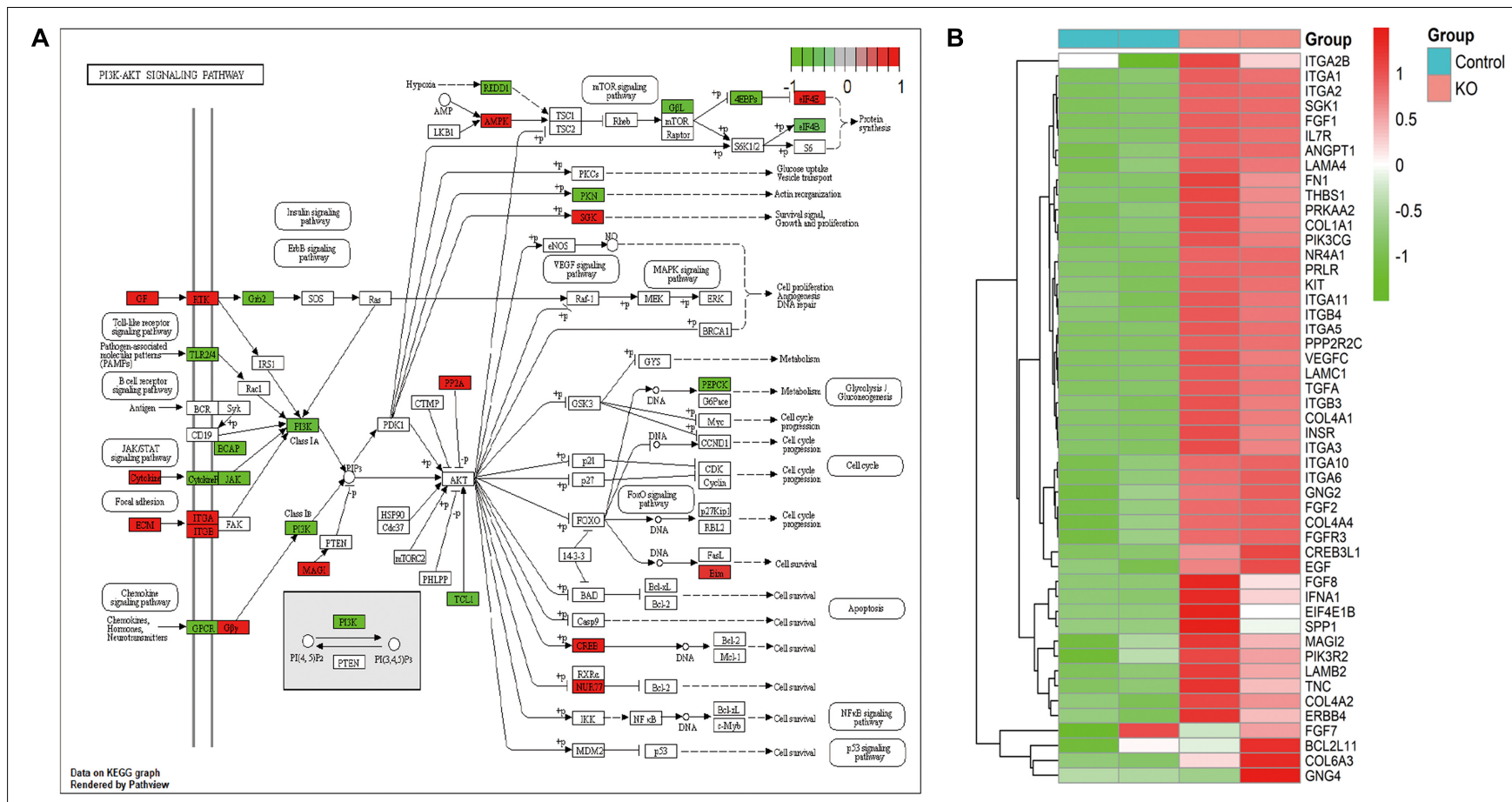

FIGURE 3 | PI3K-Akt signaling pathway for DEGs from knocking out U11. (A) PI3K-Akt signaling pathway diagram. (B) Heatmap from DEGs in this pathway.

their impact on tumor stage using TCGA database. Interestingly, overall survival (OS) of patients with bladder cancer with high FN1 expression was significantly lower than that with low expression $(P=0.012)$, while the OS of bladder cancer patients with low RPL22L1 expression was significantly lower than that of patients with high expression $(P=0.034)$ (Figure 7A). The other four genes had no significant effect on the prognosis of bladder cancer patients. The six hub genes also have an impact on bladder cancer stage. The expression of FN1, TIMP1, TGFB2, and RPL22L1 in Stage II was significantly lower than that in Stage III $(P<0.05)$, while the other two genes were not statistically different in stage (Figure 7B).

\section{DISCUSSION}

SnRNAs are a class of non-coding RNAs whose length ranges from 100 to 215 nucleotides in mammals, mainly including U1, U2, U3, U4, U5, U6, and U11 genes. SnRNAs have been present in the nucleus of mammalian cells, and together with more than 40 intranuclear proteins form RNA spliceosomes (Dvinge et al., 2019; Suzuki et al., 2019), which catalyze the maturation of precursor mRNAs in mammals, thereby affecting gene expression and leading to proliferation or apoptosis of cancer cells. We found that small nuclear RNAs mediate the formation of long-range chromatin interactions, of which U11 (RNU11) may be the most significant small nuclear RNA. To explore the gene expression changes and its potential effects mediated by U11 snRNA in bladder cancer cells, U11 snRNA knockout and overexpressed cell lines were constructed and further used to analyze the gene expression changes by RNA sequencing. Interestingly, we found that both up-regulated genes in the U11 overexpression group and down-regulated genes in the U11 knockout group were mainly enriched in cancer-related pathways, such as NFkappa B signaling pathway, TNF signaling pathway and Bladder cancer. Protein interaction network analysis predicted that CXC chemokine family (CXCL2, CXCL3, CXCL6, and CXCL8) were hub genes. Further alternative splicing analysis also found that both the U11 knockout group and the U11 overexpression group caused alternative splicing events in genes with different expression, including some genes in the PI3K-Akt signaling pathway, such as FN1 and FGF1 genes, and other oncogenes, such as TGFB2, TIMP1, and MDM2. Our results suggest that U11 may affect the expression of cancer-related genes and be implicated in bladder carcinogenesis by affecting alternative splicing.

NF-kappa B is a heterodimer composed mainly of p65 and P50 proteins, and its function is to induce the transcription factors of inflammatory cytokines and anti-apoptotic proteins. In most cells, NF-kappa B mediates cell survival signals and protects cells from apoptosis (Jung and Dritschilo, 2001). Increasing evidence suggests that activation of NF-kappa B is associated with apoptosis, expression of angiogenic proteins, and carcinogenesis due to its fundamental effects on the dedifferentiation and proliferation of malignant tumor cells (Dorai and Aggarwal, 2004; Umezawa, 2006). Related studies have found that NF-kappa $B$ activation is associated with urogenital cancers, such as prostate cancer and renal cell carcinoma (Oya et al., 2003; Ross et al., 2004; Domingo-Domenech et al., 2005). Similarly, in bladder tumors, the effect of NF-kappa B activation on tumorigenesis has also been reported (Levidou et al., 2008) and in our study, both 
A

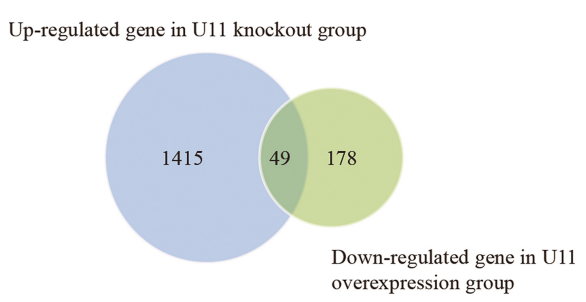

C

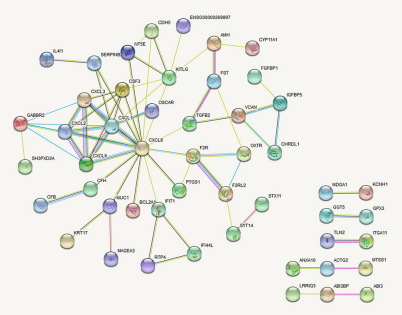

$\mathbf{F}$

G
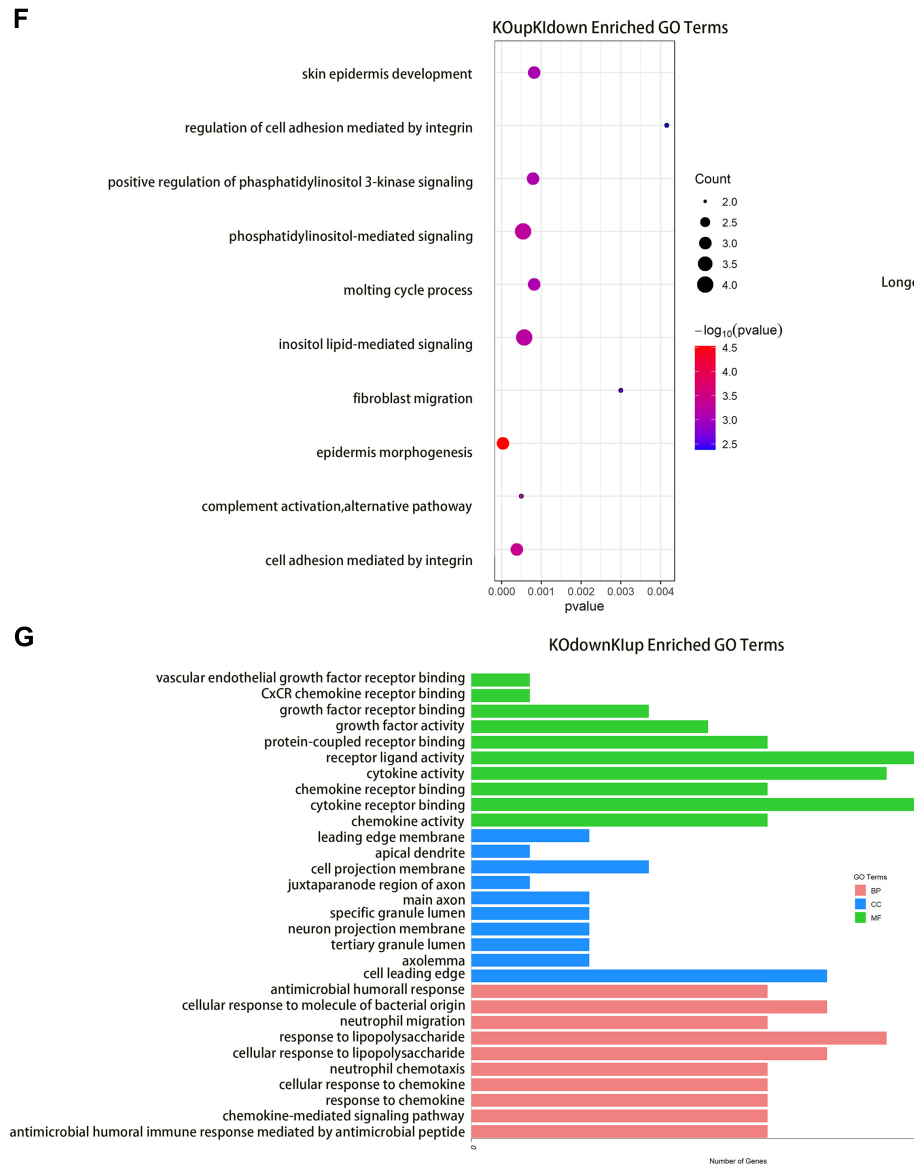

KOdownKlup Enriched GO Terms
B Down-regulated gene in U11 knockout group

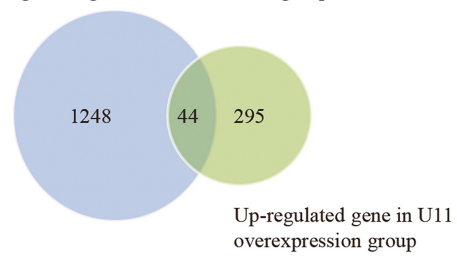

D

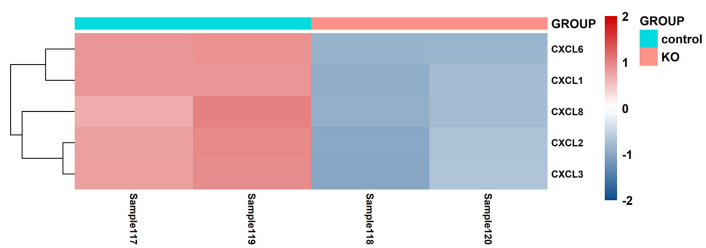

E
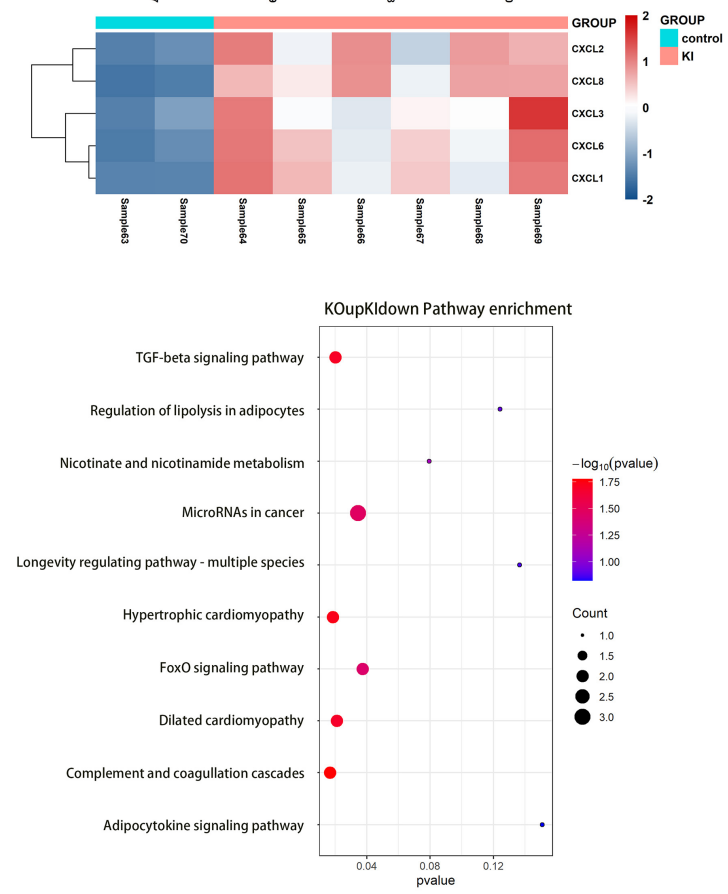

KOdownKlup Pathway enrichment

Transcriptional misregulation incanter

TNF signaling pathway

Taurine and hypotaurine metabolism

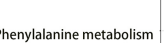

thogenic Escherichia coli infectio

NF-kappa B signaling pathway

Legionellosis $\bullet$

Hematopoietic cell lineage

Glutathione metabolism

Arachidonic acid metabolism

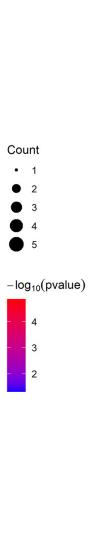

FIGURE 4 | Comprehensive analysis of DEGs upon knocking out and overexpressing U11 in T24 cell lines. (A,B) Venn diagrams of the intersection of differentially expressed genes between U11 overexpression group and knockout group. (C) Protein-protein interactions (PPI) of intersection gene. (D) The heatmap of CXCL family genes in $\mathrm{T} 24^{\mathrm{U} 11-\mathrm{KO}}$ and $\mathrm{T} 24^{\mathrm{WT}}$ cell lines. (E) The heatmap of $\mathrm{CXCL}$ family genes in $\mathrm{T} 24^{\mathrm{U} 11-\mathrm{Kl}}$ and $\mathrm{T} 24^{\mathrm{WT}}$ cell lines. (F) Pathways from intersecting genes of up-regulated genes in $\mathrm{T} 24^{\mathrm{U} 11-\mathrm{KO}}$ cell line and down-regulated genes in $\mathrm{T} 24^{\mathrm{U} 11-\mathrm{KI}}$ cell line. (G) Pathways from intersecting genes of down-regulated genes in $\mathrm{T} 24^{\mathrm{WT}}$ cell line and up-regulated genes in $\mathrm{T} 24^{\mathrm{U} 11-\mathrm{KI}}$ cell line. 

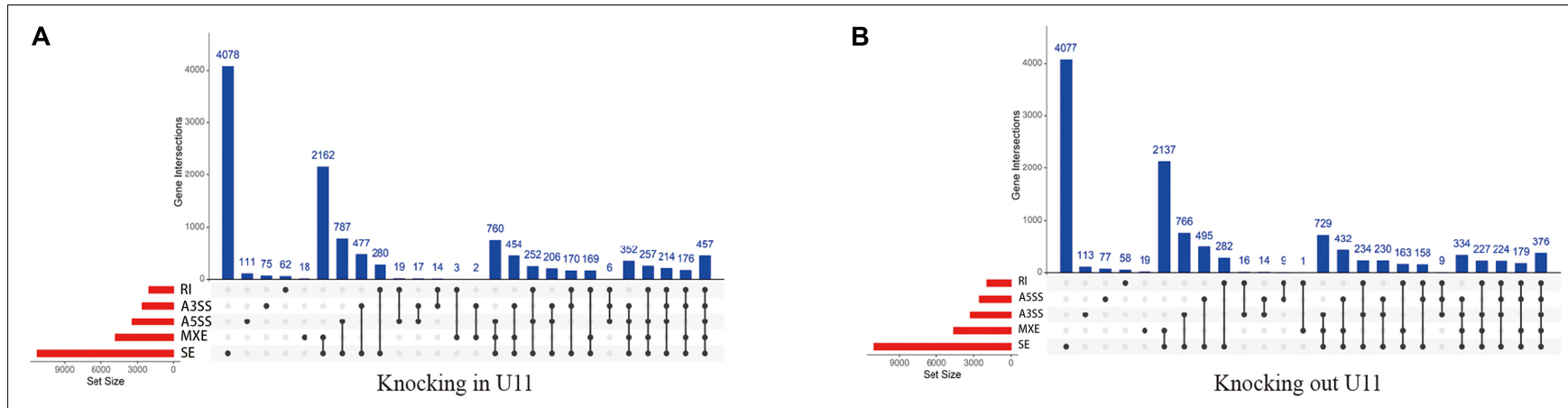

FIGURE 5 | The identification of alternative splicing events. (A,B) Up-set plots of significant alternative splicing events upon knockout and overexpressed U11 in T24 cells. Skipped exon (SE), alternative 5' splice site (A5SS), alternative $3^{\prime}$ splice site (A3SS), mutually exclusive exons (MXE), and retained intron (RI).

up-regulated genes in the U11 overexpression group and downregulated genes in the $\mathrm{U} 11$ knockout group were mainly enriched in the NF-kappa B signaling pathway.

PI3K-Akt pathway is a downstream signal transduction pathway of various cytokines and growth factors, which is involved in regulating cell proliferation and apoptosis (Lim and Counter, 2005; Bleau et al., 2009). PI3K belongs to the phospholipid kinase family and can be activated by many extracellular factors to participate in the cellular response. Activated PI3K phosphorylates PIP2-PIP3, thereby activating its downstream target kinase Akt. Activated Akt is ectopic from the cell membrane to the nucleus and cytoplasm. It activates or inhibits downstream target proteins, further promotes cell proliferation, apoptosis and energy metabolism, and is closely related to tumorigenesis and development (Franke et al., 2003). It was found that PI3K-Akt signaling pathway plays an important role in the occurrence, development and progression of malignant tumors, and the activation of Akt is closely related to the proliferation, migration and invasion of tumor cells (Roncolato et al., 2019; Ma et al., 2020). In this study, after knocking out U11, intersecting genes with significant differential alternative splicing and abnormal expression were mainly enriched in the PI3K-Akt signaling pathway.

Among them, fibronectin (FN) on the PI3K-Akt signaling pathway is a high molecular weight extracellular matrix glycoprotein with a molecular weight of $440 \mathrm{kDa}$. Its molecular structure contains a variety of domains, which can selectively bind to a variety of macromolecules in the extracellular matrix, such as collagen, heparin, fibrin and cell surface receptors, and play a crucial role in cell adhesion, migration, growth, differentiation and other cell overgrowth (Oya et al., 2003; Li et al., 2019). FN1 is a member of the FN family and plays a variety of biological functions in tumors, atherosclerosis, arthritis, and other diseases (Castelletti et al., 2008). Recent studies have found that FN1 is an important regulator promoting the formation and development of a variety of cancer cells, such as glioblastoma, laryngeal cancer and cutaneous squamous cell carcinoma (Jerhammar et al., 2010; Liao et al., 2018). In breast cancer, FN1 activates specific matrix metalloproteinases to promote breast cancer cell invasion and metastasis (Qian et al., 2011). It has been reported that the combination of miR-200c and FN1 can effectively inhibit the development of endometrial cancer in terms of FN1 expression in endometrial cancer cells (Howe et al., 2011). MiR-200c inhibits the expression of FNI, significantly reduces cell proliferation, and inhibits migration and invasion, suggesting that the expression of FN1 is a good indicator of the state of cancer cells. FN1 affects proliferation, senescence and apoptosis of human glioma cells through PI3KAKT signaling pathway (Liao et al., 2018). Down-regulation of FN1 can inhibit proliferation, migration and invasion, thereby inhibiting the occurrence of colorectal cancer (Cai et al., 2018). Interestingly, we found that the expression of FN1 increased significantly after the knockout of U11, and FN1 underwent several meaningful alternative splicing events, including three alternative $3^{\prime}$ splicing site (A3SS) events, one mutually exclusive exon event, and four exon skipping events. Although we examined three typical exons skipping of FN1 (EDA, EDB, and IIICS) as previously reported (Lopez-Mejia et al., 2013), no remarkably alternative splice events were detected in FN1 gene. Due to the numerous exons of FN1 and thus generating multiple alternative splicing events, prominent bands of these AS events were extremely challenged to detect using conventional PCR. More advanced testing technologies and further versus nested PCR experiments should be conducted to detect the multiple alternative splicing events.

Chemokines essentially belong to a class of small molecule proteins, whose initial role is mainly to participate in the directional chemotaxis of leukocytes to inflammatory sites. The role of chemokines and their receptors in the process of tumorigenesis and development cannot be ignored increasingly. A large number of studies have shown that the regularity of malignant tumor cell metastasis is similar to that of chemokine migration during inflammatory cell metastasis (Ha et al., 2017). CXCL8 is an important member of the chemokine family. It was first discovered by Yoshimura in 1987 in the culture supernatant of human peripheral blood mononuclear cells stimulated with lipopolysaccharide (Yoshimura et al., 1987) and formally named IL-8/NAP (IL-8 NAP neutrophil active peptide) in 1988. At present, studies have confirmed that CXCL8 is highly expressed in thyroid tumors, ovarian cancer, liver cancer, prostate cancer and many other tumors, and its role is mainly reflected in: accelerating the growth of tumor cells, enhancing the motility 
A

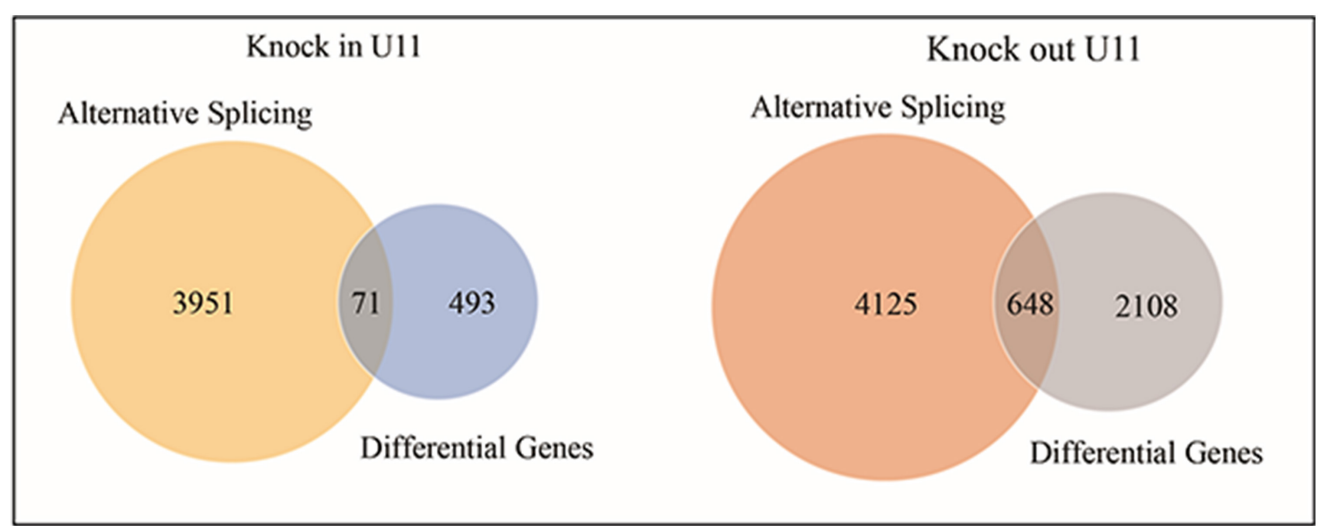

B

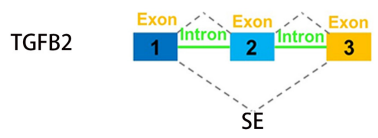

RPL22L1 11 Introǹ Exon 1 1ntron Exon 3

SE

MDM2

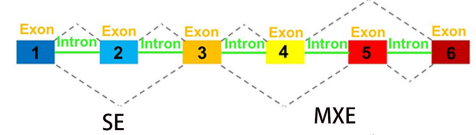

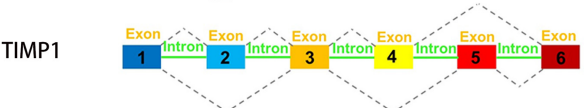

SE

MXE

FN1

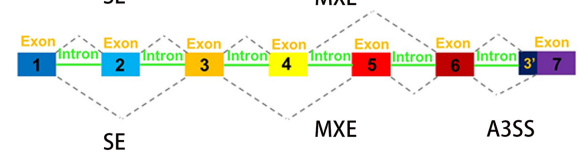

D

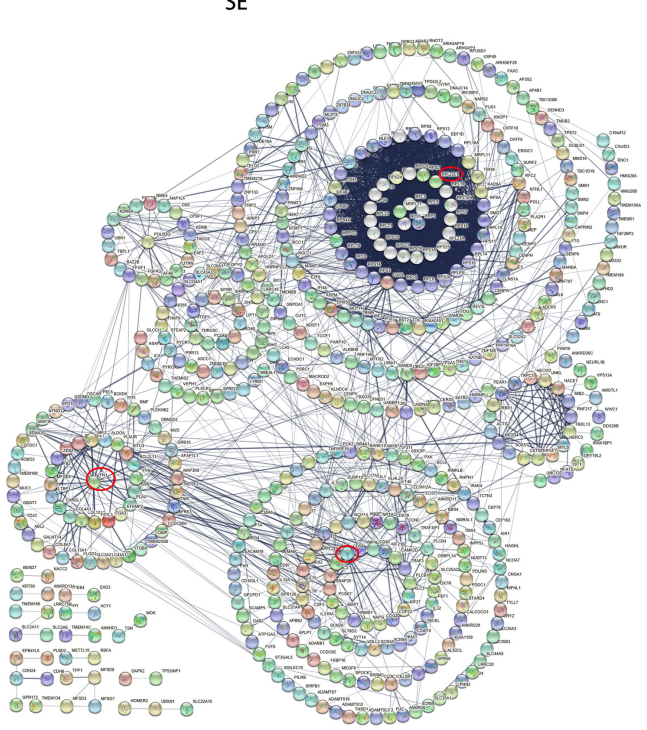

C

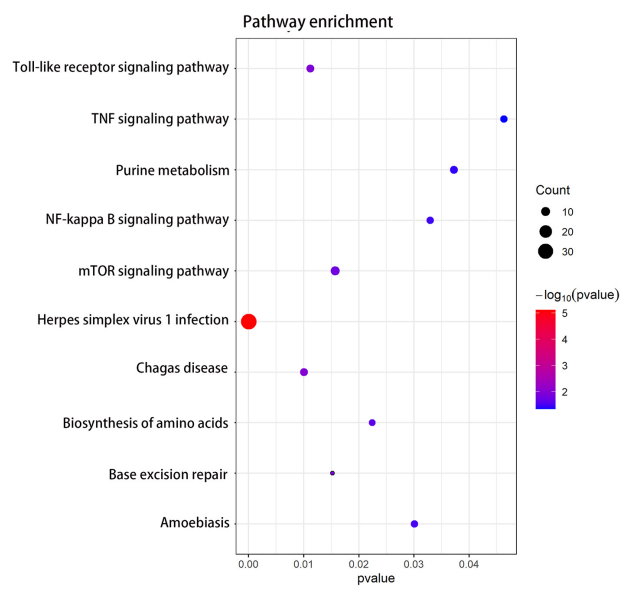

E
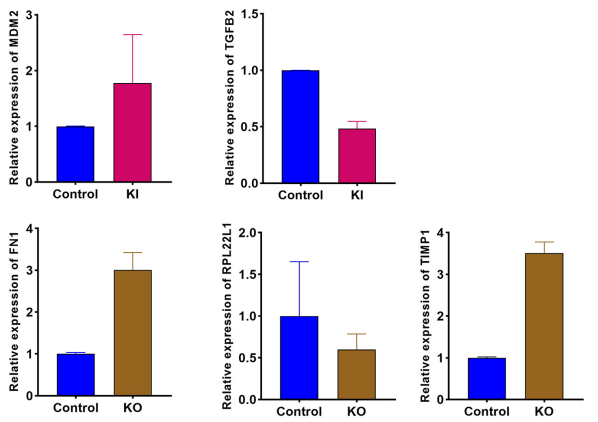

$\mathbf{F}$
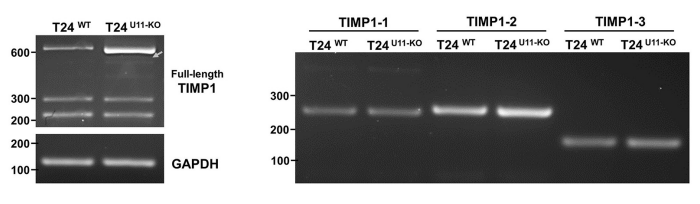

FIGURE 6 | Comprehensive analysis of alternative splicing events and differential genes upon knocking out and overexpressing U11 in T24 cell lines. (A) Venn diagrams of DEGs and genes with significant alternative splicing events. (B) Diagrams of alternative splicing events in MDM2, TGFB2, RPL22L1, FN1, and TIMP1. Mutually exclusive exons (MXE) and skipped exon (SE) in MDM2; SE in TGFB2; SE in RPL22L1; MXE and SE in TIMP1; MXE, SE and alternative 3' splice site (A3SS) in FN1. (C) Pathways from intersecting genes between T24 ${ }^{\mathrm{U} 11-\mathrm{KO}}$ and T24 ${ }^{\mathrm{WT}}$ cell lines. (D) Venn diagram of Protein-protein interactions (PPI) of intersecting genes. (E) The relative expression levels of MDM2 and TGFB2 between $\mathrm{T} 24^{\mathrm{U} 11-\mathrm{KI}}$ and $\mathrm{T} 24^{\mathrm{WT}}$ cell lines and the relative expression levels of FN1, RPL22L1, and TIMP1 between $\mathrm{T} 24^{\mathrm{U} 11-\mathrm{KO}}$ and T24 ${ }^{\mathrm{WT}}$ cell lines. (F) Alternative splice identification of TIMP1-full length, TIMP1-1, TIMP1-2, and TIMP1-3 in T24 11 -KO and T24 ${ }^{\mathrm{WT}}$ cell lines. 

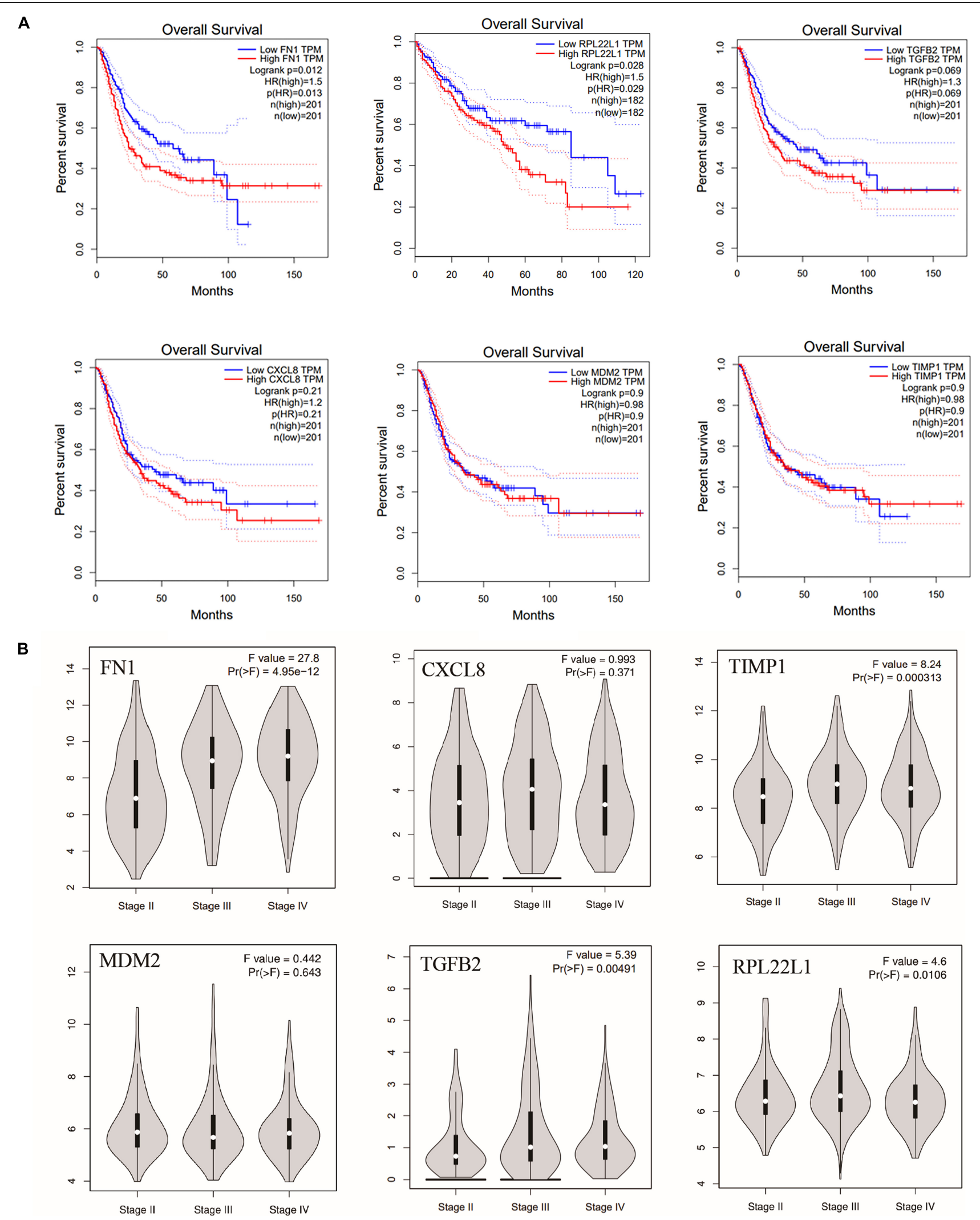

FIGURE 7 | (A) Overall survival of the six hub genes in bladder cancer based on TCGA database, including FN1, MDM2, TGFB2, CXCL8, TIMP1, and RPL22L1. OS: overall survival. (B) The effect of six hub genes on bladder cancer stage. 
of tumor cells, changing the local environment of tumors and inhibiting the immune system to play a role, and ultimately making tumor cells invade and metastasize in distant areas (Liu J. et al., 2016). In this study, we found that CXCL8 expression increased significantly after overexpression of U11, while decreased significantly after knockout of U11. Taking the intersection of the differential genes in the knockout and overexpression groups and predicting by protein interaction network analysis, we found that the chemokine family was the hub gene group, especially CXCL8. It can be seen that the expression of U11 affects the expression of the chemokine family, especially CXCL8. These results showed that the overexpression of U11 could promote the expression of chemokines, thereby promoting cell proliferation and tumor metastasis (Liu Q. et al., 2016). However, in alternative splicing analysis after knockout or overexpression of U11, we did not find significant splicing events in CXCL8, suggesting that the change in expression of U11 to CXCL8 may not be through the regulation of gene splicing. In summary, our results predict that U11 plays an important role in the regulation of chemokine expression in bladder cancer cells, but the specific mechanism is unknown.

Murine double minute 2 is a tumor protein that is highly expressed in tumors. In cancer cells, MDM2 proteins help to modify biological programs, enhance growth-promoting signals, and reduce apoptotic signals. P53 protein is a very important tumor suppressor and plays an important role in regulating cell cycle, apoptosis, DNA damage repair, angiogenesis, cell metabolism and aging (Prives, 1998; Gupta et al., 2019). In more than half of human tumors, the p53 gene is mutated or deleted, while in the remaining human tumors, there is wild-type p53, whose function is also effectively inhibited by MDM2. E3 ubiquitin ligase MDM2 is an important inhibitor of p53, which can block the transcriptional function of $\mathrm{p} 53$, promote the transfer of p53 from the nucleus to the cytoplasm, and ubiquitinate and degrade p53 (Brooks and Gu, 2006). In this study, we found that after overexpression of U11, MDM2 expression increased significantly, and MDM2 had a meaningful mutually exclusive exon and an exon skipping event. MDM2 was well-known as the most critical negative regulator of p53 pathway, whether the alternative splicing events of MDM2 directly or indirectly regulated by U11 deserved to be further investigated.

In summary, we found that U11 may alter gene expression by affecting the PI3K-Akt signaling pathway and NF-kappa B signaling pathway. U11 may be involved in the regulation of gene expression in bladder cancer cells, which may provide a novel biomarker for clinical diagnosis and treatment of bladder cancer.

\section{MATERIALS AND METHODS}

\section{Cell Lines and Cell Culture}

T24 bladder cancer cell line was purchased from Cell Bank of Shanghai Institute of Cell Biology (Shanghai, China). T24-FL and T24-SLT cell lines were gifts from Dr. Gordon Hager (NIH, United States). All cells were cultured in F12 Medium (Gibco,
China) supplemented with penicillin, streptomycin and 10\% FBS (Gibco, Australia). All cell lines were maintained at $37^{\circ} \mathrm{C}$ in a humidified atmosphere containing $5 \% \mathrm{CO}_{2}$.

\section{Design of sgRNA and Construction of Its Expression Vector}

Using an online CRISPR design tool, two sgRNAs targeting the U11 region were designed by selecting the sgRNA sequences with high scores. The sequence is as follows:

SG1-F: 5' -CACC GCTGTCGTGAGTGGCACACGT-3'

SG1-R: 5'-AAAC ACGTGTGCCACTCACGACAGC-3'

SG2-F: 5' -CACC GCAGCTGGTGATCGTTGGTCC-3'

SG2-F: 5'-AAAC GGACCAACGATCACCAGCTGC-3'

Sequencing primers were designed with the location of sgRNA as the center. The sgRNA expression vector was cloned into pX330 all in one vector by the BbsI digestion site so that the vector could express CAS9 protein and corresponding sgRNA.

\section{Cell Transfection}

$1.5 \times 10^{5}$ T24 cells/well were plated on 24-well plates and transfected after $12-16 \mathrm{~h}$ (500 ng for each of the two sgRNA vectors; sgRNA vector was used as the control group). After 6$8 \mathrm{~h}$, the liquid was changed. $48 \mathrm{~h}$ after transfection, $2 \mathrm{ug} / \mathrm{ml}$ Puro was added into the fresh medium for screening for $48 \mathrm{~h}$. Cells were then grown in a fresh medium at $37^{\circ} \mathrm{C}$ in a humidified incubator containing $5 \mathrm{c} / \mathrm{o} \mathrm{CO} 2$ and collected $24 \mathrm{~h}$ later. The cells were subjected to genome extraction, and the other part of the cells were cloned in 96-well plates.

\section{Knockout of Small Nuclear RNA U11 in T24 Bladder Cancer Cells Using CRISPR/Cas9 Gene-Editing Technology}

Genomic DNA was extracted with Tiangen Genome Extraction Kit and PCR was performed with sequencing primers. Three monoclonal cell lines were selected, and total RNA was extracted by the Tiangen RNA extraction kit for reverse transcription. Subsequently, the expression of U11 in wild-type and U11knockout cells was detected by fluorescence quantitative PCR, and the knockout efficiency of U11 was identified. The monoclonal cell lines with the highest knockout efficiency were selected to construct the U11 knockout model.

\section{Construction of Stable Overexpressing U11 Bladder Cancer T24 Cells by pcDNA-U11 Recombinant Plasmid Transfection}

RNA from T24 cells was extracted and reverse transcribed into cDNA. Using this cDNA as a template, the U11 gene sequence was amplified with specific PCR primers. The U11 fragment and pcDNA3 were digested by HindIII and KpnI restriction endonucleases. The product was ligated by T4 DNA ligase and transformed into E. coli DH5 $\alpha$ cells. The plasmid identified by sequencing was named pcDNA-U11. After transfection, identification, and monoclonal selection, the culture was expanded. 


\section{Cell Proliferation Assay}

$\mathrm{T} 24^{\mathrm{WT}}$ and $\mathrm{T} 24^{\mathrm{U} 11-\mathrm{KO}}$ cells in logarithmic growth phase were digested and seeded into 96-well plates at the concentration of 3,500 cells per well. For the MTT assay, cells were cultured for $0,24,48,72$, and $96 \mathrm{~h}$, respectively and then $10 \mathrm{ul} \mathrm{MTT}$ $(5 \mathrm{mg} / \mathrm{mL})$ was added into each well for another $4 \mathrm{~h}$ at $37^{\circ} \mathrm{C}$. MTT solution was then removed, and MTT formazan dissolved in $100 \mu \mathrm{L}$ dimethyl sulfoxide (DMSO) for detection of the absorbance at $490 \mathrm{~nm}$.

\section{Immunofluorescence}

To detect expression of CBs at cellular levels, IF localization was conducted according to standard procedures. First, cells were fixed with $4 \%$ paraformaldehyde for $20 \mathrm{~min}$ at room temperature and permeabilized with $0.2 \%$ Triton $\mathrm{X}-100$ for $10 \mathrm{~min}$ on ice. Then, cells were washed three times with PBS. Subsequently, cells were incubated with anti-coilin antibody (Cat\# 10967-1-AP, Proteintech, United States) for $1 \mathrm{~h}$, and followed by incubation with secondary antibody (Goat Anti-Mouse IgG, DyLight 488). Cells were then counterstained with DAPI after washing three times with PBS. Multicolor imaging was performed and captured utilizing an IX70 microscope at $20 \times$ magnification (Olympus, Japan).

\section{mRNA Library Construction and Sequencing}

A total of 12 samples were taken for RNA sequencing, including 6 overexpressed U11 samples (sample64-smaple69), 2 knockout U11 samples (sample118, sample120), and 4 control samples (sample63, sample70, sample117, and sample119). Total RNA was extracted from the control group, U11 knockout bladder cancer cells, and U11 overexpressing bladder cancer cells cultured in vitro. The ribosome RNA was removed by ribosome RNA depletion kit and then reverse transcribed into cDNA for second-strand synthesis. dsDNA is interrupted by ultrasound to grow uniform fragments. The fragments were flattened, $3^{\prime} \mathrm{A}$ bases were generated, and the adaptor was ligated to complete the construction of the RNA-seq high-throughput sequencing library. Highthroughput sequencing via Illumina HiSeq2000 platform. All operations were performed by Shanghai WUXI NEXTCODE. Sequencing was performed using the Illumina system, following the protocol provided by Illumina, with $2 \times 150$ pairedend sequencing.

\section{The Analysis of Alternative Splicing}

After comparing the data, the file is converted to bam format using Samtools. Then alternative splice analysis was performed using rMATS 4.0.2. rMATS is a software for differential alternative splice analysis of RNA-seq data. The rMATS statistical model was used to quantify the expression of alternative splice events in different samples, and then the $P$-value was calculated by the Likelihood Ratio Test to represent the differences in LncLevel (Inclusion Level) between the two groups of samples. There are five kinds of alternative splice events recognized by rMATS, respectively skipped exon (SE), alternative 5 splice site (A5SS), A3SS, mutually exclusive exons (MXE), retained intron (RI) (Shen et al., 2014). The detailed results of alternative splicing in T24 U11-KI and T24 U11-KO cell lines are presented in Supplementary Materials.

\section{RNA Extraction, PCR, and DNA Agarose GEL Electrophoresis}

Total RNA was extracted from the control group, U11 knockout bladder cancer cells, and U11 overexpressing bladder cancer cells cultured in vitro using Trizol Reagent (Invitrogen), and reverse-transcribed to cDNA using PrimeSciptTM RT reagent Kit with Gdna Eraser (Takara, China) following the manufacturer's instructions. Primer sequence were displayed in Table 1.

PCR was performed in 20 ul reactions containing 10 ul $2 \times$ ES Taq MasterMix (CW BIO, China), $3.4 \mathrm{ul} \mathrm{H}_{2} \mathrm{O}, 0.8 \mathrm{ul}$ of each gene-specific primer and $5 \mathrm{ul}$ cDNA. Reaction conditions were 30 cycles of $94^{\circ} \mathrm{C}$ for $2 \mathrm{~min}, 60^{\circ} \mathrm{C}$ for 30 s and $72^{\circ} \mathrm{C}$ for $30 \mathrm{~s}$. PCR products were separated by $3 \mathrm{c} / \mathrm{o}$ gel electrophoresis. Then Image Quant LAS 500 was used for exposure.

\section{Data Processing and Bioinformatics Analysis}

Sequencing data is Illumina raw data of RNA-seq. Fastqc is used to evaluate the quality of raw data. Fastp is used for data pre-processing, including removing adapter components and effectively correcting lower quality bases. After fastp treatment, Fastqc detects data quality again and obtains qualified clean data. Clean data were aligned to the reference genome hg38 using Hisat2, gene expression was obtained by Stringtie, and differential genes were finally obtained by the Deseq 2 $\mathrm{R}$ package (|Fold change $\geq 1.5$ and $P<0.05$ ). Gene ontology (GO) and The Kyoto Encyclopedia of Genes and Genomes (KEGG) pathway enrichment analysis were used for DEGs using the clusterProfiler $\mathrm{R}$ package, and significant enrichment was defined as $P<0.05$. Cytoscape was used to construct the U11 regulatory network. The prognostic analysis of core genes was performed using GEPIA based on the TCGA database.

TABLE 1 | Primers used for PCR.

\begin{tabular}{lll}
\hline Gene & Forward primer $\mathbf{5}^{\prime} \sim \mathbf{3}^{\prime}$ & Reverse primer $\mathbf{5}^{\prime} \sim \mathbf{3}^{\prime}$ \\
\hline TIMP1 & CCCTAGCGTGGACATTATC & AAGGTGACGGGACTGGAAG \\
Full-length & & \\
TIMP1-1 & CCCTAGCGTGGACATTATC & GGTATAAGGTGGTCTGGTTG \\
TIMP1-2 & ACTTCCACAGGTCCCACAAC & AAGGTGACGGGACTGGAAG \\
TIMP1-3 & CTTCTGGCATCCTGTTGTG & GGTATAAGGTGGTCTGGTTG \\
GAPDH & GTGAACCATGAGAAGTAT & CATGAGTCCTCCACGATACC \\
& GACAAC & \\
SnRNA U11 & AGATAGGTAATACGACTCAC & TTAACCCTCACTAAAGG \\
& TATAG & GAAGAA \\
& GGAAAAAGGGCTTCTGTC & AGGGCGCCGGGACC \\
& GTGAGTG &
\end{tabular}




\section{DATA AVAILABILITY STATEMENT}

The datasets presented in this study can be found in the Gene Expression Omnibus database (GEO) under accession number: GSE171744.

\section{AUTHOR CONTRIBUTIONS}

QW and ZT conceived the study. YX, ZW, and XW wrote the manuscript. YW, ST, CF, and LP analyzed the results. QL and YT edited the manuscript. All authors contributed to the article and approved the submitted version.

\section{REFERENCES}

Andrade, L. E., Chan, E. K., Raska, I., Peebles, C. L., Roos, G., and Tan, E. M. (1991). Human autoantibody to a novel protein of the nuclear coiled body: immunological characterization and cDNA cloning of p80-coilin. J. Exp. Med. 173, 1407-1419. doi: 10.1084/jem.173.6.1407

Bleau, A. M., Hambardzumyan, D., Ozawa, T., Fomchenko, E. I., Huse, J. T., Brennan, C. W., et al. (2009). PTEN/PI3K/Akt pathway regulates the side population phenotype and ABCG2 activity in glioma tumor stem-like cells. Cell Stem Cell 4, 226-235. doi: 10.1016/j.stem.2009.01.007

Bray, F., Ferlay, J., Soerjomataram, I., Siegel, R. L., Torre, L. A., and Jemal, A. (2018). Global cancer statistics 2018: GLOBOCAN estimates of incidence and mortality worldwide for 36 cancers in 185 countries. CA Cancer J. Clin. 68, 394-424. doi: 10.3322/caac.21492

Brooks, C. L., and Gu, W. (2006). p53 ubiquitination: Mdm2 and beyond. Mol. Cell 21, 307-315. doi: 10.1016/j.molcel.2006.01.020

Cai, X., Liu, C., Zhang, T. N., Zhu, Y. W., Dong, X., and Xue, P. (2018). Down-regulation of FN1 inhibits colorectal carcinogenesis by suppressing proliferation, migration, and invasion. J. Cell Biochem. 119, 4717-4728. doi: $10.1002 /$ jcb. 26651

Castelletti, F., Donadelli, R., Banterla, F., Hildebrandt, F., Zipfel, P. F., Bresin, E., et al. (2008). Mutations in FN1 cause glomerulopathy with fibronectin deposits. Proc. Natl. Acad. Sci. U.S.A. 105, 2538-2543. doi: 10.1073/pnas.0707730105

Domingo-Domenech, J., Mellado, B., Ferrer, B., Truan, D., Codony-Servat, J., Sauleda, S., et al. (2005). Activation of nuclear factor-kappaB in human prostate carcinogenesis and association to biochemical relapse. Br. J. Cancer 93, 12851294. doi: $10.1038 /$ sj.bjc. 6602851

Dorai, T., and Aggarwal, B. B. (2004). Role of chemopreventive agents in cancer therapy. Cancer Lett. 215, 129-140. doi: 10.1016/j.canlet.2004.07.013

Dvinge, H., Guenthoer, J., Porter, P. L., and Bradley, R. K. (2019). RNA components of the spliceosome regulate tissue- and cancer-specific alternative splicing. Genome Res. 29, 1591-1604. doi: 10.1101/gr.246678.118

Feng, R. M., Zong, Y. N., Cao, S. M., and Xu, R. H. (2019). Current cancer situation in China: good or bad news from the 2018 Global Cancer Statistics? Cancer Commun. 39:22. doi: 10.1186/s40880-019-0368-6

Franke, T. F., Hornik, C. P., Segev, L., Shostak, G. A., and Sugimoto, C. (2003). PI3K/Akt and apoptosis: size matters. Oncogene 22, 8983-8998. doi: 10.1038/sj. onc. 1207115

Gupta, A., Behl, T., Heer, H. R., Deshmukh, R., and Sharma, P. L. (2019). Mdm2-P53 interaction inhibitor with cisplatin enhances apoptosis in colon and prostate cancer cells In-Vitro. Asian Pac. J. Cancer Prev. 20, 3341-3351. doi: 10.31557/APJCP.2019.20.11.3341

Ha, H., Debnath, B., and Neamati, N. (2017). Role of the CXCL8-CXCR1/2 Axis in Cancer and inflammatory diseases. Theranostics 7, 1543-1588. doi: 10.7150/ thno. 15625

Hearst, S. M., Gilder, A. S., Negi, S. S., Davis, M. D., George, E. M., Whittom, A. A., et al. (2009). Cajal-body formation correlates with differential coilin phosphorylation in primary and transformed cell lines. J. Cell Sci. 122(Pt 11), 1872-1881. doi: $10.1242 /$ jcs. 044040

\section{FUNDING}

This work was supported by the National Natural Science Foundation of China (82060512, 82060460, and 31471271), the Guangxi Natural Science Fund for Innovation Research Team (2016GXNSFGA380006), and Guangxi Science and Technology Base and Talent Special Fund (2019AC17009 and AD17195090).

\section{SUPPLEMENTARY MATERIAL}

The Supplementary Material for this article can be found online at: https://www.frontiersin.org/articles/10.3389/fgene. 2021.695597/full\#supplementary-material

Hebert, M. D. (2010). Phosphorylation and the Cajal body: modification in search of function. Arch. Biochem. Biophys. 496, 69-76. doi: 10.1016/j.abb.2010.02.012

Hebert, M. D. (2013). Signals controlling Cajal body assembly and function. Int. J. Biochem. Cell Biol. 45, 1314-1317. doi: 10.1016/j.biocel.2013.03.019

Howe, E. N., Cochrane, D. R., and Richer, J. K. (2011). Targets of miR-200c mediate suppression of cell motility and anoikis resistance. Breast Cancer Res. 13:R45. doi: $10.1186 /$ bcr 2867

Jeppesen, D. K., Nawrocki, A., Jensen, S. G., Thorsen, K., Whitehead, B., Howard, K. A., et al. (2014). Quantitative proteomics of fractionated membrane and lumen exosome proteins from isogenic metastatic and nonmetastatic bladder cancer cells reveal differential expression of EMT factors. Proteomics 14, 699712. doi: 10.1002/pmic.201300452

Jerhammar, F., Ceder, R., Garvin, S., Grénman, R., Grafström, R. C., and Roberg, K. (2010). Fibronectin 1 is a potential biomarker for radioresistance in head and neck squamous cell carcinoma. Cancer Biol. Ther. 10, 1244-1251. doi: $10.4161 / \mathrm{cbt} .10 .12 .13432$

Jung, M., and Dritschilo, A. (2001). NF-kappa B signaling pathway as a target for human tumor radiosensitization. Semin. Radiat. Oncol. 11, 346-351. doi: 10.1053/srao.2001.26034

Levidou, G., Saetta, A. A., Korkolopoulou, P., Papanastasiou, P., Gioti, K., Pavlopoulos, P., et al. (2008). Clinical significance of nuclear factor (NF)kappaB levels in urothelial carcinoma of the urinary bladder. Virchows. Arch. 452, 295-304. doi: 10.1007/s00428-007-0560-y

Li, B., Shen, W., Peng, H., Li, Y., Chen, F., Zheng, L., et al. (2019). Fibronectin 1 promotes melanoma proliferation and metastasis by inhibiting apoptosis and regulating EMT. Onco Targets Ther. 12, 3207-3221. doi: 10.2147/OTT.S195703

Liang, Z., Mou, Q., Pan, Z., Zhang, Q., Gao, G., Cao, Y., et al. (2019). Identification of candidate diagnostic and prognostic biomarkers for human prostate cancer: RPL22L1 and RPS21. Med. Oncol. 36:56. doi: 10.1007/s12032-019-1283-z

Liao, Y. X., Zhang, Z. P., Zhao, J., and Liu, J. P. (2018). Effects of fibronectin 1 on cell proliferation, senescence and apoptosis of human glioma cells through the PI3K/AKT signaling pathway. Cell Physiol. Biochem. 48, 1382-1396. doi: $10.1159 / 000492096$

Lim, K. H., and Counter, C. M. (2005). Reduction in the requirement of oncogenic Ras signaling to activation of PI3K/AKT pathway during tumor maintenance. Cancer Cell 8, 381-392. doi: 10.1016/j.ccr.2005.10.014

Liu, J., Xu, R., and Zhao, X. (2016). [Mechanisms for effect of osthole on inhibiting the growth and invasion of bladder cancer cells]. Zhong Nan Da Xue Xue Bao Yi Xue Ban 41, 345-352. doi: 10.11817/j.issn.1672-7347.2016.04.002

Liu, Q., Li, A., Tian, Y., Wu, J. D., Liu, Y., Li, T., et al. (2016). The CXCL8CXCR1/2 pathways in cancer. Cytokine Growth Factor Rev. 31, 61-71. doi: 10.1016/j.cytogfr.2016.08.002

Lopez-Mejia, I. C., De Toledo, M., Della Seta, F., Fafet, P., Rebouissou, C., Deleuze, V., et al. (2013). Tissue-specific and SRSF1-dependent splicing of fibronectin, a matrix protein that controls host cell invasion. Mol. Biol. Cell 24, 3164-3176. doi: $10.1091 / \mathrm{mbc}$.E13-03-0142

Lui, L., and Lowe, T. (2013). Small nucleolar RNAs and RNA-guided posttranscriptional modification. Essays Biochem. 54, 53-77. doi: 10.1042/ bse 0540053 
Ma, Z., Yu, R., Zhu, Q., Sun, L., Jian, L., Wang, X., et al. (2020). CXCL16/CXCR6 axis promotes bleomycin-induced fibrotic process in MRC-5 cells via the PI3K/AKT/FOXO3a pathway. Int. Immunopharmacol. 81:106035. doi: 10.1016/ j.intimp.2019.106035

Machyna, M., Heyn, P., and Neugebauer, K. M. (2013). Cajal bodies: where form meets function. Wiley Interdiscip. Rev. RNA 4, 17-34. doi: 10.1002/wrna.1139

Nicholson, B. E., Frierson, H. F., Conaway, M. R., Seraj, J. M., Harding, M. A., Hampton, G. M., et al. (2004). Profiling the evolution of human metastatic bladder cancer. Cancer Res. 64, 7813-7821. doi: 10.1158/0008-5472.Can-040826

Oya, M., Takayanagi, A., Horiguchi, A., Mizuno, R., Ohtsubo, M., Marumo, K., et al. (2003). Increased nuclear factor-kappa B activation is related to the tumor development of renal cell carcinoma. Carcinogenesis 24, 377-384. doi: 10.1093/ carcin/24.3.377

Ploeg, M., Aben, K. K., and Kiemeney, L. A. (2009). The present and future burden of urinary bladder cancer in the world. World J. Urol. 27, 289-293. doi: 10.1007/s00345-009-0383-3

Prives, C. (1998). Signaling to p53: breaking the MDM2-p53 circuit. Cell 95, 5-8. doi: 10.1016/s0092-8674(00)81774-2

Qian, P., Zuo, Z., Wu, Z., Meng, X., Li, G., Wu, Z., et al. (2011). Pivotal role of reduced let-7g expression in breast cancer invasion and metastasis. Cancer Res. 71, 6463-6474. doi: 10.1158/0008-5472.CAN-11-1322

Roncolato, F., Lindemann, K., Willson, M. L., Martyn, J., and Mileshkin, L. (2019). $\mathrm{PI} 3 \mathrm{~K} / \mathrm{AKT} / \mathrm{mTOR}$ inhibitors for advanced or recurrent endometrial cancer. Cochrane Database Syst. Rev. 10:CD012160. doi: 10.1002/14651858.CD012160. pub2

Ross, J. S., Kallakury, B. V., Sheehan, C. E., Fisher, H. A., Kaufman, R. P. Jr., Kaur, P., et al. (2004). Expression of nuclear factor-kappa B and I kappa B alpha proteins in prostatic adenocarcinomas: correlation of nuclear factor-kappa B immunoreactivity with disease recurrence. Clin. Cancer Res. 10, 2466-2472. doi: 10.1158/1078-0432.ccr-0543-3

Salim, E. I., Moore, M. A., Bener, A., Habib, O. S., Seif-Eldin, I. A., and Sobue, T. (2010). Cancer epidemiology in South-West Asia - past, present and future. Asian Pac. J. Cancer Prev. 11(Suppl. 2), 33-48.

Shen, S., Park, J. W., Lu, Z. X., Lin, L., Henry, M. D., Wu, Y. N., et al. (2014). rMATS: robust and flexible detection of differential alternative splicing from replicate RNA-Seq data. Proc. Natl. Acad. Sci. U.S.A. 111, E5593-E5601. doi: 10.1073/pnas.1419161111

Strzelecka, M., Trowitzsch, S., Weber, G., Lührmann, R., Oates, A. C., and Neugebauer, K. M. (2010). Coilin-dependent snRNP assembly is essential for zebrafish embryogenesis. Nat. Struct. Mol. Biol. 17, 403-409. doi: 10.1038/nsmb. 1783

Suzuki, H., Kumar, S. A., Shuai, S., Diaz-Navarro, A., Gutierrez-Fernandez, A., De Antonellis, P., et al. (2019). Recurrent noncoding U1 snRNA mutations drive cryptic splicing in SHH medulloblastoma. Nature 574, 707-711. doi: 10.1038/ s41586-019-1650-0

Umezawa, K. (2006). Inhibition of tumor growth by NF-kappaB inhibitors. Cancer Sci. 97, 990-995. doi: 10.1111/j.1349-7006.2006.00285.x

Usher, P. A., Sieuwerts, A. M., Bartels, A., Lademann, U., Nielsen, H. J., HoltenAndersen, L., et al. (2007). Identification of alternatively spliced TIMP-1 mRNA in cancer cell lines and colon cancer tissue. Mol. Oncol. 1, 205-215. doi: 10.1016/ j.molonc.2007.05.002

Wang, Q., Sawyer, I. A., Sung, M. H., Sturgill, D., Shevtsov, S. P., Pegoraro, G., et al. (2016). Cajal bodies are linked to genome conformation. Nat. Commun. 7:10966. doi: 10.1038/ncomms10966

Yoshimura, T., Matsushima, K., Oppenheim, J. J., and Leonard, E. J. (1987). Neutrophil chemotactic factor produced by lipopolysaccharide (LPS)-stimulated human blood mononuclear leukocytes: partial characterization and separation from interleukin 1 (IL 1). J. Immunol. 139, $788-793$.

Conflict of Interest: The authors declare that the research was conducted in the absence of any commercial or financial relationships that could be construed as a potential conflict of interest.

Copyright (c) 2021 Wang, Wang, Wang, Tang, Feng, Pan, Lu, Tao, Xie, Wang and Tang. This is an open-access article distributed under the terms of the Creative Commons Attribution License (CC BY). The use, distribution or reproduction in other forums is permitted, provided the original author(s) and the copyright owner(s) are credited and that the original publication in this journal is cited, in accordance with accepted academic practice. No use, distribution or reproduction is permitted which does not comply with these terms. 